Journal of Mathematical Physics, Analysis, Geometry

2017, Vol. 13, No. 2, pp. 154-172

doi: $10.15407 / \operatorname{mag} 13.02 .154$

\title{
Homogenized Model of Non-Stationary Diffusion in Porous Media with the Drift
}

\author{
M. Goncharenko \\ B. Verkin Institute for Low Temperature Physics and Engineering \\ of the National Academy of Sciences of Ukraine \\ 47 Nauky Ave., Kharkiv 61103, Ukraine \\ E-mail: marusya61@yahoo.co.uk \\ L. Khilkova \\ Institute of Chemical Technology of Eastern Ukrainian National University \\ 31 Volodymyrska Str., Rubizhne 93009, Ukraine \\ E-mail: LarisaHilkova@gmail.com
}

Received June 1, 2016, revised November 16, 2016

\begin{abstract}
We consider an initial boundary-value problem for a parabolic equation describing non-stationary diffusion in porous media with non-linear absorption on the boundary and the transfer of the diffusing substance by fluid. We prove the existence of the unique solution for this problem. We study the asymptotic behavior of a sequence of solutions when the scale of microstructure tends to zero and obtain the homogenized model of the diffusion process.
\end{abstract}

Key words: homogenization, non-stationary diffusion, non-linear boundary condition, homogenized model.

Mathematics Subject Classification 2010: 35Q74.

\section{Introduction}

Homogenization problems for partial differential equations have been studied by mathematicians for over 50 years and many significant results have been obtained (see, for example, [1, 3, 4, 7, 18, 24-26, 28, 30, 35]. These problems appear in the study of physical processes in a strongly inhomogeneous medium. The scale of microstructure is very small, so the properties of the medium change rapidly in space. Therefore the coefficients of the equations describing these processes are changed rapidly by the space coordinates. It is practically impossible to solve the boundary or initial-boundary value problems for these equations either analytically or numerically. When the scale of microstructure tends to zero,

(c) M. Goncharenko and L. Khilkova, 2017 
the problem can be studied by using the asymptotic analysis which leads to a homogenized (macroscopic) model of the corresponding processes.

In recent years, more attention has been paid to non-linear problems. In particular, there is a number of publications which study the stationary and non-stationary diffusion in a porous medium with non-linear absorption on the boundary $[2,5,6,10,11,20-23,27,31-34]$. All these publications consider periodic porous media consisting of absorbing solid grains arranged periodically in space. From a physical point of view, it is more natural to consider a medium consisting of a connected absorbing solid phase which is not necessarily periodic.

In the present paper, we study the asymptotic behavior of the solution of the initial boundary-value problem for a parabolic equation. It describes the process of non-stationary diffusion in a porous medium with non-linear absorption on the boundary and the transfer of the diffused impurity by fluid flowing in the pores. The system of pores is assumed to be a domain that satisfies the condition of the strong connectivity (see definition in Sec. 2). For periodically perforated domains, the equation with the linear Robin condition was studied in [7-9] and for the strongly connected domains with linear absorption in [4]. The problem of a stationary diffusion without drift is studied in [12], and the result is used in this paper.

The paper is organized as follows. In Sec. 2, the statement of the problem and the qualitative description of the results (homogenized model) are given. In Sec. 3, we prove the existence and uniqueness of the solutions of the initial problem. In Sec. 4, we define the local characteristics of the porous medium. In Sec. 5, we obtain the uniform estimates for the solution of the initial problem, and in Sec. 6, we prove the main theorem on the convergence of the solution of the initial problem to the solution of the homogenized problem when the scale of microstructure tends to zero.

\section{Statement of the Problem}

Let $\Omega$ be a domain in $R^{n}(n \geq 2)$ with a smooth boundary $\partial \Omega, F^{\varepsilon}$ be a closed set in $\Omega$ depending on a small parameter $\varepsilon$ such that $F^{\varepsilon}$ becomes more porous and more dense in $\Omega$ as $\varepsilon \rightarrow 0$. We assume that the boundary of $F^{\varepsilon}$ is smooth.

Consider the initial boundary-value problem in $\Omega^{\varepsilon} \times(0, T)$, where $\Omega^{\varepsilon}=\Omega \backslash$ $F^{\varepsilon}$,

$$
\begin{gathered}
\frac{\partial u^{\varepsilon}(x, t)}{\partial t}-\Delta u^{\varepsilon}(x, t)+\sum_{i=1}^{n} v_{i}^{\varepsilon}(x) \frac{\partial u^{\varepsilon}(x, t)}{\partial x_{i}}=0 \text { in } \Omega^{\varepsilon} \times(0, T) \\
\frac{\partial u^{\varepsilon}}{\partial \nu}+\sigma^{\varepsilon}\left(x, u^{\varepsilon}\right)=0 \text { on } \partial F^{\varepsilon} \times(0, T) \\
u^{\varepsilon}(x, t)=0 \text { on } \partial \Omega \times(0, T)
\end{gathered}
$$




$$
u^{\varepsilon}(x, 0)=\varphi(x) \text { in } \Omega^{\varepsilon},
$$

where $\Delta=\sum_{i=1}^{n} \frac{\partial^{2}}{\partial x_{i}^{2}}$ is the Laplace operator, $\nu$ is the exterior unit normal with respect to $\partial F^{\varepsilon}$; the functions $\varphi(x), \sigma^{\varepsilon}(x, s)$ and a vector-function $v^{\varepsilon}(x)$ are given.

Problem (1)-(4) describes a diffusion of particles in a porous medium. The last term in the left-hand side of (1) means the transfer of particles by fluid while (2) describes the absorption on the boundary. The velocity of the fluid is denoted by $v^{\varepsilon}(x)=\left\{v_{1}^{\varepsilon}(x), v_{2}^{\varepsilon}(x), \ldots, v_{n}^{\varepsilon}(x)\right\}$.

We assume that the function $\sigma^{\varepsilon}(x, s)$ satisfies the conditions:

$\left.a_{1}\right) \sigma^{\varepsilon}(x, s) \in L^{\infty}\left(\partial \Omega^{\varepsilon} ; C^{1}\left(R^{1}\right)\right), \quad \sigma^{\varepsilon}(x, 0)=0 ;$

$\left.a_{2}\right) 0<\frac{\partial}{\partial s} \sigma^{\varepsilon}(x, s) \leq \hat{\sigma}^{\varepsilon}(x)$, where the function $\hat{\sigma}^{\varepsilon}(x)$ satisfies the condition that for every ball $B(\rho, z)$ of radius $\rho$ with a center in a point $z \in \Omega$ the inequality

$$
\int_{\partial F^{\varepsilon} \cap B(\rho, z)} \hat{\sigma}^{\varepsilon}(x) d \Gamma<C_{1} \rho^{n}+C_{2}(\varepsilon) \rho^{n-1}
$$

holds, where the constant $C_{1}$ is independent of $z, \rho$ and $\varepsilon$, the constant $C_{2}(\varepsilon)$ is independent of $z, \rho$ and $C_{2}(\varepsilon) \rightarrow 0$ as $\varepsilon \rightarrow 0$.

$\mathrm{R}$ e $\mathrm{m}$ a $\mathrm{r} \mathrm{k}$ 1. The last condition ensures that the density of the absorption at the surface of solid phase becomes small in mean as $\varepsilon \rightarrow 0$. The smallness of the absorption is explained with a simple example. Consider a periodic location of the particles. The particles are the balls with radius of order $\varepsilon$ that form a periodic lattice with the period of the same order. If the absorption function is taken in the form $\sigma^{\varepsilon}(x, s)=\sigma(\varepsilon) s$, then the $\hat{\sigma}^{\varepsilon}(x)=\sigma(\varepsilon)$. The radius of the ball $B(\rho, z)$ can be much greater than $\varepsilon$ or smaller than $\varepsilon$, or of order $\varepsilon$. If $\rho \gg \varepsilon$, then the surface integral in $\left.a_{2}\right)$ is of order $\sigma(\varepsilon) \varepsilon^{-1} \rho^{n}$. If $\rho \leq \varepsilon$, then the surface integral in $a_{2}$ ) is of order $\sigma(\varepsilon) \rho^{n-1}$. For condition $a_{2}$ ) to be satisfied, it is necessary that $\sigma(\varepsilon) \sim \varepsilon$. Thus the density of the absorption at the surface of solid phase becomes small.

We consider the case where the transfer is carried out by an ideal incompressible fluid. The vector-function $v^{\varepsilon}(x)$ satisfies the conditions:

$\left.b_{1}\right) v_{i}^{\varepsilon}(x) \in H^{1}\left(\Omega^{\varepsilon}\right),\left.v_{\nu}^{\varepsilon}\right|_{F^{\varepsilon}}=0$, where $v_{\nu}^{\varepsilon}$ is a normal component of the velocity vector $v^{\varepsilon}$;

$\left.b_{2}\right) \operatorname{div} v^{\varepsilon}=0, x \in \Omega^{\varepsilon}$

b3) $\max _{x \in \Omega^{\varepsilon}}\left|v_{i}^{\varepsilon}(x)\right| \leq C$, where $C$ does not depend on $\varepsilon$. 
We assume that the following inequality holds for any $\varepsilon>0$ :

$$
\operatorname{mes}\left(\Omega^{\varepsilon}\right) \geq C_{1}>0
$$

and $\Omega^{\varepsilon}$ satisfies the extension condition. Namely, for any function $\vartheta^{\varepsilon}(x) \in H^{1}\left(\Omega^{\varepsilon}\right)$ there exists a function $\tilde{\vartheta}^{\varepsilon}(x) \in H^{1}(\Omega)$ such that $\vartheta^{\varepsilon}(x)=\tilde{\vartheta}^{\varepsilon}(x)$ for $x \in \Omega^{\varepsilon}$, and the inequality

$$
\left\|\nabla \tilde{\vartheta}^{\varepsilon}\right\|_{L^{2}(\Omega)} \leq C_{2}\left\|\nabla \vartheta^{\varepsilon}\right\|_{L^{2}\left(\Omega^{\varepsilon}\right)}
$$

holds, where the constants $C_{1}, C_{2}$ are independent of $\varepsilon$.

$\mathrm{R}$ e $\mathrm{m}$ a $\mathrm{r} \mathrm{k} 2$. It is easy to see $[18, \mathrm{p} .150]$ that the following inequality implies from (5), (6):

$$
\left\|\tilde{\vartheta}^{\varepsilon}\right\|_{H^{1}(\Omega)} \leq C\left\|\vartheta^{\varepsilon}\right\|_{H^{1}\left(\Omega^{\varepsilon}\right)},
$$

where the constant $C$ does not depend on $\varepsilon$. Thus, the domains $\Omega^{\varepsilon}$ satisfy the strong connectivity condition.

Our main goal is to study the asymptotic behavior of the solution $u^{\varepsilon}(x, t)$ of problem (1)-(4) as $\varepsilon \rightarrow 0$. We show that under certain conditions a sequence $\left\{u^{\varepsilon}(x, t)\right\}$ converges to the solution of the homogenized problem

$$
\begin{gathered}
b(x) \frac{\partial u}{\partial t}-\sum_{i, k=1}^{n} \frac{\partial}{\partial x_{i}}\left(a_{i k}(x) \frac{\partial u}{\partial x_{k}}\right)+\sum_{i=1}^{n} v_{i}(x) \frac{\partial u}{\partial x_{i}}+\frac{1}{2} c_{u}(x, u)=0, \\
\text { in } \Omega \times(0, T), \\
u(x, t)=0, \text { on } \partial \Omega \times(0, T), \\
u(x, 0)=\varphi(x), \text { in } \Omega .
\end{gathered}
$$

Here $b(x)$ is the volume density of the porous medium, $c_{u}(x, u)$ is the limit density of the absorption, $a(x)$ is the conductivity tensor of the medium, $v(x)$ is the vector of the limit velocity of the drift.

Before formulating the main theorem, we will prove the existence and uniqueness of the weak solution of the initial problem for each fixed $\varepsilon$.

\section{The Existence and Uniqueness of the Weak Solution of Problem (1)-(4)}

Let us introduce the functional spaces:

$$
\begin{aligned}
& H^{1}\left(\Omega^{\varepsilon}, \partial \Omega\right)=\left\{u \in H^{1}\left(\Omega^{\varepsilon}\right):\left.u\right|_{\partial \Omega}=0\right\}, \\
& W^{2}\left(0, T ; \Omega^{\varepsilon}, \partial \Omega\right)=\left\{u \in L^{2}\left(0, T ; H^{1}\left(\Omega^{\varepsilon}, \partial \Omega\right)\right), u_{t}^{\prime} \in L^{2}\left(0, T ; H^{1}\left(\Omega^{\varepsilon}, \partial \Omega\right)^{*}\right)\right\} .
\end{aligned}
$$


The weak solution of problem (1)-(4) is defined as follows.

A function $u^{\varepsilon} \in W^{2}\left(0, T ; \Omega^{\varepsilon}, \partial \Omega\right)$ is a weak solution of problem (1)-(4) if for any function $\vartheta(x) \in H^{1}\left(\Omega^{\varepsilon}, \partial \Omega\right)$ the following identity holds for almost all $t \in$ $(0, T)$ and $\left.u^{\varepsilon}\right|_{t=0}=\varphi(x)$,

$$
\left\langle\left(u^{\varepsilon}\right)_{t}^{\prime}, \vartheta\right\rangle+\int_{\Omega^{\varepsilon}}\left(\nabla u^{\varepsilon} \nabla \vartheta+\sum_{i=1}^{n} v_{i}^{\varepsilon}(x) \frac{\partial u^{\varepsilon}}{\partial x_{i}} \vartheta\right) d x+\int_{\partial F^{\varepsilon}} \sigma^{\varepsilon}\left(x, u^{\varepsilon}\right) \vartheta d \Gamma=0 .
$$

Here $\left\langle\left(u^{\varepsilon}\right)_{t}^{\prime}, \vartheta\right\rangle$ is the action of the functional $\left(u^{\varepsilon}\right)_{t}^{\prime} \in H^{1}\left(\Omega^{\varepsilon}, \partial \Omega\right)^{*}$ on an element $\vartheta(x) \in H^{1}\left(\Omega^{\varepsilon}, \partial \Omega\right)$.

This definition is correct as $\varphi(x) \in L^{2}\left(\Omega^{\varepsilon}\right)$. Since $W^{2}\left(0, T ; \Omega^{\varepsilon}, \partial \Omega\right)$ is embedded in $C\left([0, T] ; L^{2}\left(\Omega^{\varepsilon}\right)\right)$ according to Proposition 1.2 [29, p. 106], the equality $\left.u^{\varepsilon}\right|_{t=0}=\varphi(x)$ has a sense.

Theorem 1. Let the function $\varphi(x) \in H^{1}\left(\Omega^{\varepsilon}, \partial \Omega\right)$, the function $\sigma^{\varepsilon}(x, s)$ satisfy the conditions $\left.\left.a_{1}\right)-a_{2}\right)$, and the vector-function $v^{\varepsilon}(x)$ satisfy the conditions $\left.\left.b_{1}\right)-b_{3}\right)$. Then problem (1)-(4) has a unique weak solution $u^{\varepsilon} \in W^{2}\left(0, T ; \Omega^{\varepsilon}, \partial \Omega\right)$ for every fixed $\varepsilon$.

P r o o f. We define an operator $A: H^{1}\left(\Omega^{\varepsilon}, \partial \Omega\right) \rightarrow H^{1}\left(\Omega^{\varepsilon}, \partial \Omega\right)^{*}$ for almost all $t \in(0, T)$ as follows:

$$
\langle A u, \vartheta\rangle=\int_{\Omega^{\varepsilon}}\left(\nabla u^{\varepsilon} \nabla \vartheta+\sum_{i=1}^{n} v_{i}^{\varepsilon}(x) \frac{\partial u^{\varepsilon}}{\partial x_{i}} \vartheta\right) d x+\int_{\partial F^{\varepsilon}} \sigma^{\varepsilon}\left(x, u^{\varepsilon}\right) \vartheta d \Gamma, \vartheta \in H^{1}\left(\Omega^{\varepsilon}, \partial \Omega\right) .
$$

In the same way as in Proposition 2.1 [29, p. 108], one can prove that the function $u^{\varepsilon} \in W^{2}\left(0, T ; \Omega^{\varepsilon}, \partial \Omega\right)$ is the weak solution of problem (1)-(4) if and only if it is a solution of the abstract Cauchy problem

$$
\left\{\begin{array}{l}
\left(u^{\varepsilon}\right)_{t}^{\prime}+A u^{\varepsilon}=0 \text { in } L^{2}\left(0, T ; H^{1}\left(\Omega^{\varepsilon}, \partial \Omega\right)^{*}\right) \\
u^{\varepsilon}(0)=\varphi \text { in } H^{1}\left(\Omega^{\varepsilon}, \partial \Omega\right)
\end{array}\right.
$$

In view of the isomorphism $L^{2}\left(0, T ; H^{1}\left(\Omega^{\varepsilon}, \partial \Omega\right)\right)^{*} \cong L^{2}\left(0, T ; H^{1}\left(\Omega^{\varepsilon}, \partial \Omega\right)^{*}\right)$, the operator $A: H^{1}\left(\Omega^{\varepsilon}, \partial \Omega\right) \rightarrow H^{1}\left(\Omega^{\varepsilon}, \partial \Omega\right)^{*}$ generates its realization $\mathcal{A}: L^{2}(0, T$; $\left.H^{1}\left(\Omega^{\varepsilon}, \partial \Omega\right)\right) \rightarrow L^{2}\left(0, T ; H^{1}\left(\Omega^{\varepsilon}, \partial \Omega\right)^{*}\right)$ which has basic properties similar to those of the operator $A$.

Let us prove the necessary properties of the operator $A: H^{1}\left(\Omega^{\varepsilon}, \partial \Omega\right) \rightarrow$ $H^{1}\left(\Omega^{\varepsilon}, \partial \Omega\right)^{*}$.

The operator $A$ is bounded. Using the Cauchy-Bunyakovsky inequality and 
the properties of the functions $v^{\varepsilon}(x)$ and $\sigma^{\varepsilon}(x, s)$, we obtain

$$
\begin{aligned}
& \left|\left\langle A u^{\varepsilon}, \vartheta\right\rangle\right| \leq\left|\int_{\Omega^{\varepsilon}} \nabla u^{\varepsilon} \nabla \vartheta d x\right|+\sum_{i=1}^{n}\left|\int_{\Omega^{\varepsilon}} v_{i}^{\varepsilon}(x) \frac{\partial u^{\varepsilon}}{\partial x_{i}} \vartheta d x\right|+\left|\int_{\partial F^{\varepsilon}} \sigma^{\varepsilon}\left(x, u^{\varepsilon}\right) \vartheta d \Gamma\right| \\
& \leq\left\|\nabla u^{\varepsilon}\right\|_{L^{2}\left(\Omega^{\varepsilon}\right)}\|\nabla \vartheta\|_{L^{2}\left(\Omega^{\varepsilon}\right)}+C_{1} \sum_{i=1}^{n}\left\|\frac{\partial u^{\varepsilon}}{\partial x_{i}}\right\|_{L^{2}\left(\Omega^{\varepsilon}\right)}\|\vartheta\|_{L^{2}\left(\Omega^{\varepsilon}\right)}+C_{2}\left|\int_{\partial F^{\varepsilon}} \hat{\sigma}^{\varepsilon}(x) u^{\varepsilon} \vartheta d \Gamma\right| \\
& \leq\left(1+C_{1}\right)\left\|u^{\varepsilon}\right\|_{H^{1}\left(\Omega^{\varepsilon}, \partial \Omega\right)}\|\vartheta\|_{H^{1}\left(\Omega^{\varepsilon}, \partial \Omega\right)}+C_{2}\left|\int_{\partial F^{\varepsilon}} \hat{\sigma}^{\varepsilon}(x)^{1 / 2} u^{\varepsilon} \hat{\sigma}^{\varepsilon}(x)^{1 / 2} \vartheta d \Gamma\right| \\
& \leq\left(1+C_{1}\right)\left\|u^{\varepsilon}\right\|_{H^{1}\left(\Omega^{\varepsilon}, \partial \Omega\right)}\|\vartheta\|_{H^{1}\left(\Omega^{\varepsilon}, \partial \Omega\right)}+C_{2}\left\|u^{\varepsilon}\right\|_{L^{2}\left(\Omega, \mu^{\varepsilon}\right)}\|\vartheta\|_{L^{2}\left(\Omega, \mu^{\varepsilon}\right)},
\end{aligned}
$$

where $L^{2}\left(\Omega, \mu^{\varepsilon}\right)$ is the space with a measure $d \mu^{\varepsilon}=\hat{\sigma}^{\varepsilon}(x) d \Gamma$.

Due to the property $\left.a_{2}\right)$ of the function $\sigma^{\varepsilon}(x, s)$, the measure $\mu^{\varepsilon}$ satisfies

$$
\int_{\partial F^{\varepsilon} \cap B(\rho, z)} d \mu^{\varepsilon}<C \rho^{n-1} .
$$

Then, according to the generalized Sobolev theorem $\left[19\right.$, p. 58], the space $H^{1}(\Omega)$ is embedded in the space $L^{2}\left(\Omega, \mu^{\varepsilon}\right)$. Finally, using this theorem and inequality (7), we obtain

$$
\left\|A u^{\varepsilon}\right\|_{H^{1}\left(\Omega^{\varepsilon}, \partial \Omega\right)^{*}}=\sup _{\|\vartheta\| \leq 1}\left|\left\langle A u^{\varepsilon}, \vartheta\right\rangle\right| \leq \tilde{C}\left\|u^{\varepsilon}\right\|_{H^{1}\left(\Omega^{\varepsilon}, \partial \Omega\right)} .
$$

The operator $A$ is monotone. In view of the properties of the functions $v^{\varepsilon}(x)$ and $\sigma^{\varepsilon}(x, s)$, we have

$$
\begin{aligned}
& \langle A u-A \vartheta, u-\vartheta\rangle=\int_{\Omega^{\varepsilon}}|\nabla(u-\vartheta)|^{2} d x+\frac{1}{2} \sum_{i=1}^{n} \int_{\Omega^{\varepsilon}} v_{i}^{\varepsilon}(x) \frac{\partial(u-\vartheta)^{2}}{\partial x_{i}} d x \\
& +\int_{\partial F^{\varepsilon}}\left(\sigma^{\varepsilon}(x, u)-\sigma^{\varepsilon}(x, \vartheta)\right)(u-\vartheta) d \Gamma=\int_{\Omega^{\varepsilon}}|\nabla(u-\vartheta)|^{2} d x \\
& +\int_{\partial F^{\varepsilon}}\left(\sigma^{\varepsilon}(x, u)-\sigma^{\varepsilon}(x, \vartheta)\right)(u-\vartheta) d \Gamma \geq 0 .
\end{aligned}
$$

The operator $A$ is hemicontinuous. For each $u, \vartheta \in H^{1}\left(\Omega^{\varepsilon}, \partial \Omega\right)$, the real valued function

$$
t \mapsto\langle A(u+t \vartheta), \vartheta\rangle=\int_{\Omega^{\varepsilon}}\left(\nabla(u+t \vartheta) \nabla \vartheta+\sum_{i=1}^{n} v_{i}^{\varepsilon}(x) \frac{\partial(u+t \vartheta)}{\partial x_{i}} \vartheta\right) d x
$$




$$
+\int_{\partial F^{\varepsilon}} \sigma^{\varepsilon}(x, u+t \vartheta) \vartheta d \Gamma
$$

is continuous with respect to $t$. The continuity of the first term is obvious. The second term is continuous with respect to $t$ due to the properties of the function $\sigma^{\varepsilon}(x, s)$ as shown below:

$$
\begin{aligned}
& \left|\int_{\partial F^{\varepsilon}} \sigma^{\varepsilon}\left(x, u+t_{1} \vartheta\right) \vartheta d \Gamma-\int_{\partial F^{\varepsilon}} \sigma^{\varepsilon}\left(x, u+t_{2} \vartheta\right) \vartheta d \Gamma\right|=\left|\int_{\partial F^{\varepsilon}} \int_{u+t_{1} \vartheta}^{u+t_{2} \vartheta} \frac{\partial \sigma^{\varepsilon}(x, s)}{\partial s} d s \vartheta d \Gamma\right| \\
& \leq\left|t_{1}-t_{2}\right| \int_{\partial F^{\varepsilon}} \hat{\sigma}^{\varepsilon}(x) \vartheta^{2} d \Gamma=\left|t_{1}-t_{2}\right| \cdot\|\vartheta\|_{L^{2}\left(\Omega, \mu^{\varepsilon}\right)}^{2} \leq C_{1}\left|t_{1}-t_{2}\right| \cdot\|\vartheta\|_{H^{1}\left(\Omega^{\varepsilon}, \partial \Omega\right)}^{2} \\
& \leq C_{2}\left|t_{1}-t_{2}\right| .
\end{aligned}
$$

Thus we have proved that the operator $A$ is monotone, hemicontinuous and bounded, and by virtue of Lemma $4.2[29$, p. 122], the realization $\mathcal{A}$ : $L^{2}\left(0, T ; H^{1}\left(\Omega^{\varepsilon}, \partial \Omega\right)\right) \rightarrow L^{2}\left(0, T ;\left(H^{1}\left(\Omega^{\varepsilon}, \partial \Omega\right)\right)^{*}\right)$ is monotone, hemicontinuous and bounded. It means that $\mathcal{A}$ is of type $M$ (Lemma $2.1[29$, p. 38]).

Let us show that the realization $\mathcal{A}: L^{2}\left(0, T ; H^{1}\left(\Omega^{\varepsilon}, \partial \Omega\right)\right) \rightarrow$ $L^{2}\left(0, T ;\left(H^{1}\left(\Omega^{\varepsilon}, \partial \Omega\right)\right)^{*}\right)$ is a coercive operator. Indeed, using the properties of the functions $v^{\varepsilon}(x)$ and $\sigma^{\varepsilon}(x, s)$, and the strong connectivity of the domains $\Omega^{\varepsilon}(7)$, for every function $\vartheta \in L^{2}\left(0, T ; H^{1}\left(\Omega^{\varepsilon}, \partial \Omega\right)\right)$ and its extension $\tilde{\vartheta} \in L^{2}\left(0, T ; \stackrel{\circ}{H}^{1}(\Omega)\right)$, we have

$$
\begin{aligned}
& \langle\mathcal{A} \vartheta, \vartheta\rangle=\int_{0}^{T}\langle A \vartheta, \vartheta\rangle d t=\int_{0}^{T} \int_{\Omega^{\varepsilon}}|\nabla \vartheta|^{2} d x d t+\frac{1}{2} \sum_{i=1}^{n} \int_{0}^{T} \int_{\Omega^{\varepsilon}} v_{i}^{\varepsilon}(x) \frac{\partial \vartheta^{2}}{\partial x_{i}} d x d t \\
& +\int_{0}^{T} \int_{\partial F^{\varepsilon}} \sigma^{\varepsilon}(x, \vartheta) \vartheta d \Gamma d t=\int_{0}^{T} \int_{\Omega^{\varepsilon}}|\nabla \vartheta|^{2} d x d t+\int_{0}^{T} \int_{\partial F^{\varepsilon}} \sigma^{\varepsilon}(x, \vartheta) \vartheta d \Gamma \\
& \geq \int_{0}^{T} \int_{\Omega^{\varepsilon}}^{T}|\nabla \vartheta|^{2} d x d t \geq C \int_{0}^{T} \int_{\Omega}|\nabla \tilde{\vartheta}|^{2} d x d t=C \int_{0}^{T}\|\tilde{\vartheta}\|_{H^{1}(\Omega)}^{2} d t \\
& \geq C \int_{0}^{T}\|\vartheta\|_{H^{1}\left(\Omega^{\varepsilon}, \partial \Omega\right)}^{2} d t=C\|\vartheta\|_{L^{2}\left(0, T ; H^{1}\left(\Omega^{\varepsilon}, \partial \Omega\right)\right)}^{2} .
\end{aligned}
$$

Thus,

$$
\frac{\langle\mathcal{A} \vartheta, \vartheta\rangle}{\|\vartheta\|} \geq C\|\vartheta\| \rightarrow \infty \text { as }\|\vartheta\| \rightarrow \infty
$$

where $\|\vartheta\|=\|\vartheta\|_{L^{2}\left(0, T ; H^{1}\left(\Omega^{\varepsilon}, \partial \Omega\right)\right)}$. So, $\mathcal{A}$ is coercive operator. 
Applying Theorem 4.1 [29, p. 123], we can conclude that the Cauchy problem (12) has a solution which is also a weak solution of problem (1)-(4). Its uniqueness follows directly from the monotony of $A$. Assume that the initial boundary-value problem has two different solutions $u_{1}^{\varepsilon}(x, t)$ and $u_{2}^{\varepsilon}(x, t)$. Then from the definition of $A$ and (11), we have

$$
\frac{d}{d t}\left\|u_{1}^{\varepsilon}-u_{2}^{\varepsilon}\right\|_{L^{2}\left(\Omega^{\varepsilon}\right)}^{2}=-2\left\langle A u_{1}^{\varepsilon}-A u_{2}^{\varepsilon}, u_{1}^{\varepsilon}-u_{2}^{\varepsilon}\right\rangle \leq 0, \quad 0 \leq t \leq T .
$$

Consequently, they are necessarily equal on $[0, T]$, and $u_{1}^{\varepsilon}(x, 0)=u_{2}^{\varepsilon}(x, 0)$.

Theorem 1 is proved.

\section{Local Characteristics of the Microstructure of Domains $\Omega^{\varepsilon}$ and the Convergence Theorem}

First we define local characteristics of the porous medium.

Let $K_{h}^{z}=K(z, h)$ be a cube with side $h(0<\varepsilon \ll h \ll 1)$ and center at the point $z$. The sides of the cube are oriented by the coordinate axes. The size of the cube is much greater than the characteristic scale of the microstructure $\varepsilon$, but much smaller than the size of the domain $\Omega$, so it can be called mezocube. We describe the structure of the domain $\Omega^{\varepsilon}$ with the help of the conductivity tensor $a_{i k}(z, \varepsilon, h)$ and with the help of the absorption function $c(z, s ; \varepsilon, h)$.

Consider the functional with respect to an arbitrary vector $\ell \in R^{n}$,

$$
T_{h, z}^{\varepsilon}(\ell)=\inf _{\vartheta^{\varepsilon}} \int_{K_{h}^{z} \cap \Omega^{\varepsilon}}\left(\left|\nabla \vartheta^{\varepsilon}\right|^{2}+h^{-2-\tau}\left|\vartheta^{\varepsilon}-(x-z, \ell)\right|^{2}\right) d x,
$$

where the infimum is taken over all functions $\vartheta^{\varepsilon}(x) \in H^{1}\left(K_{h}^{z} \cap \Omega^{\varepsilon}\right), \tau \in(0,2)$ is a penalty parameter. This functional is a homogeneous quadratic function of $\ell$ (see [18, p. 179]):

$$
T_{h, z}^{\varepsilon}(\ell)=\sum_{i, k=1}^{n} a_{i k}(z, \varepsilon, h) \ell_{i} \ell_{k},
$$

where the coefficients $a_{i k}(z, \varepsilon, h)$ are defined by the formula

$$
\begin{aligned}
& a_{i k}(z, \varepsilon, h)= \\
& =\int_{K_{h}^{z} \cap \Omega^{\varepsilon}}\left\{\left(\nabla \vartheta_{i}^{\varepsilon}, \nabla \vartheta_{k}^{\varepsilon}\right)+h^{-2-\tau}\left[\vartheta_{i}^{\varepsilon}-\left(x_{i}-z_{i}\right)\right]\left[\vartheta_{k}^{\varepsilon}-\left(x_{k}-z_{k}\right)\right]\right\} d x,
\end{aligned}
$$

$\vartheta_{i}^{\varepsilon}$ is the function that minimizes (13) where $\ell=e^{i}$ is the unit vector of the axis $x_{i}$. 
It follows from (14) and (15) that the tensor $\left\{a_{i k}(z, \varepsilon, h)\right\}_{i, k=1}^{n}$ is symmetric and positive definite in $R^{n}$. We call it the conductivity tensor of the domains $\Omega^{\varepsilon}$.

Consider the functional with respect to an arbitrary $s \in R^{1}$,

$$
\begin{aligned}
& c(z, s ; \varepsilon, h) \\
& =\inf _{w^{\varepsilon}}\left[\int_{K_{h}^{z} \cap \Omega^{\varepsilon}}\left\{\left|\nabla w^{\varepsilon}\right|^{2}+h^{-2-\tau}\left|w^{\varepsilon}-s\right|^{2}\right\} d x+\int_{K_{h}^{z} \cap \partial F^{\varepsilon}} g^{\varepsilon}\left(x, w^{\varepsilon}\right) d \Gamma\right],
\end{aligned}
$$

where the infimum is taken over all functions $w^{\varepsilon} \in H^{1}\left(K_{h}^{z} \cap \Omega^{\varepsilon}\right)$ and a function $g^{\varepsilon}(x, s)$ is defined by the formula

$$
g^{\varepsilon}(x, s)=2 \int_{0}^{s} \sigma^{\varepsilon}(x, r) d r .
$$

A sequence of functions $\vartheta^{\varepsilon}(x) \in L^{2}\left(\Omega^{\varepsilon}\right)$ is said to converge in $L^{2}\left(\Omega^{\varepsilon}, \Omega\right)$ if there exists a function $\vartheta(x) \in L^{2}(\Omega)$ such that $\vartheta^{\varepsilon}$ converges to $\vartheta(x)$ in the norm of $L^{2}\left(\Omega^{\varepsilon}\right)$ :

$$
\left\|\vartheta^{\varepsilon}-\vartheta\right\|_{L^{2}\left(\Omega^{\varepsilon}\right)}^{2}=\int_{\Omega^{\varepsilon}}\left|\vartheta^{\varepsilon}(x)-\vartheta(x)\right|^{2} d x \rightarrow 0, \text { as } \varepsilon \rightarrow 0 .
$$

The asymptotic behavior of the solution of problem (1)-(4) as $\varepsilon \rightarrow 0$ is described by the following theorem.

Theorem 2. Let the domains $\Omega^{\varepsilon}$ be strongly connected, the function $\varphi(x) \in H^{2}(\Omega) \cap C_{0}(\Omega)$, the vector-function $v^{\varepsilon}(x)$ satisfy the conditions $\left.b_{1}\right)$ $\left.b_{3}\right)$. Suppose the sequence of $\left\{v^{\varepsilon}(x)\right\}$ extended by zero into $F^{\varepsilon}$ converges weakly in $\left(L^{2}(\Omega)\right)^{n}$ to the vector-function $v(x)$ as $\varepsilon \rightarrow 0$. Furthermore, $\exists \tau \in(0,2)$ such that the following conditions hold uniformly in $\Omega$ :

i. $\lim _{h \rightarrow 0} \lim _{\varepsilon \rightarrow 0} \frac{\operatorname{mes}\left[K(x, h) \cap \Omega^{\varepsilon}\right]}{h^{n}}=b(x), \forall x \in \Omega$, where $b(x)$ is positive and continuous in $\Omega$;

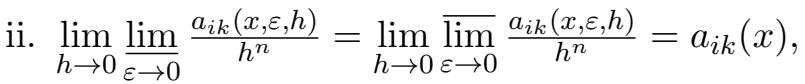
where $a_{i k}(x)$ are continuous in $\Omega$ and the tensor $\left\{a_{i k}(x)\right\}_{i, k=1}^{n}$ is symmetric positive definite in $\Omega$;

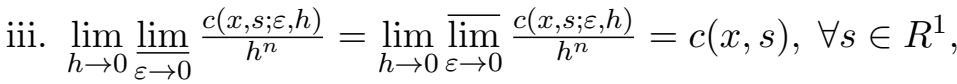
where $c(x, s)$ is bounded with respect to $x$, differentiable with respect to $s$, and its derivative $c_{s}(x, s) \equiv \frac{\partial}{\partial s} c(x, s)$ satisfies the condition

$$
\forall s \in R^{1}: 0 \leq c_{s}(x, s) \leq C .
$$


Then the sequence of weak solutions $\left\{u^{\varepsilon}(x, t)\right\}$ of problem (1)-(4) converges in $L^{2}\left(\Omega^{\varepsilon}, \Omega\right)$ to the weak solution $u(x, t)$ of the homogenized problem (8)-(10) for almost all $t \in(0, T)$.

Conditions 2) and 3) of Theorem 2 are not effective for calculating the conductivity tensor $\left\{a_{i k}(x)\right\}_{i, k=1}^{n}$ and the absorption function $c(x, s)$ for an arbitrary connected medium. However, in the simple case they can be computed explicitly.

E x a m p le 1. Let the set $F^{\varepsilon}=\bigcup_{i} F_{i}^{\varepsilon}$ be a constriction of some fixed domain $F \in K_{1}$ centered at zero with a smooth boundary $\partial F$. Here $F_{i}^{\varepsilon}=\varepsilon F+$ $x_{\varepsilon}^{i}\left(x_{\varepsilon}^{i} \in \varepsilon \mathbb{Z}^{n}\right)$, and $K_{1}$ is a unit cube. The limit characteristics of this medium can be calculated by the formulas:

$$
\begin{gathered}
b(x)=1-|F|, \\
c_{u}(x, u)=2|\partial F| \sigma(x, u), \\
a_{i k}(x)=\delta_{i k}(1-|F|)-\int_{K_{1} \backslash F} \sum_{j=1}^{n} \frac{\partial V_{i}(\xi)}{\partial \xi_{j}} \cdot \frac{\partial V_{k}(\xi)}{\partial \xi_{j}} d \xi,
\end{gathered}
$$

where $|\partial F|$ is the area of the surface $\partial F$ and $|F|$ is the volume of the domain $F$, the function $V_{k}(\xi)(k=\overline{1, n})$ is the solution of the 'cell' problem:

$$
\left\{\begin{array}{l}
\sum_{i=1}^{n} \frac{\partial^{2} V_{k}(\xi)}{\partial \xi_{i}^{2}}=0, \quad \xi \in K_{1} \backslash F \\
\frac{\partial V_{k}(\xi)}{\partial \nu_{\xi}}=\cos \left(\nu(\xi), e^{k}\right), \quad \xi \in \partial F \\
\left.V_{k}\right|_{\Gamma_{i}^{+}}=\left.V_{k}\right|_{\Gamma_{i}^{-}},\left.\quad \frac{\partial V_{k}}{\partial \xi_{i}}\right|_{\Gamma_{i}^{+}}=\left.\frac{\partial V_{k}}{\partial \xi_{i}}\right|_{\Gamma_{i}^{-}}, \quad i=\overline{1, n} \\
\int_{K_{1} \backslash F} V_{k}(\xi) d \xi=0
\end{array}\right.
$$

here $\Gamma_{i}^{ \pm}$are the opposite bounds of $K_{1}, \nu=\nu(\xi)$ is a unit normal with respect to $F$.

A more general case of a locally periodic structure of the medium was considered in [13].

The scheme of the proof of the theorem is as follows. First we obtain the estimates for the time derivative of the solution and the drift term to transform the parabolic problem to the elliptic one. Then, using Theorem 1 from [12], we obtain the homogenized model of the initial problem. 


\section{Auxiliary Estimates of the Solution $u^{\varepsilon}(x, t)$}

In the sequel, we suppose that the solution of problem (1)-(4) is smooth enough. Namely, $u^{\varepsilon} \in W^{\varepsilon}:=\left\{\forall t \in(0, T) u^{\varepsilon} \in H^{1}\left(\Omega^{\varepsilon}\right), \frac{\partial u^{\varepsilon}}{\partial t} \in L^{2}\left(0, T ; H^{1}\left(\Omega^{\varepsilon}\right)\right)\right\}$. This fact can be proved by using standard methods of improvement of the smoothness of weak solutions (see, for example, [17]).

Lemma 1. Let $\varphi(x) \in H^{2}(\Omega) \cap C_{0}(\Omega)$ and the vector-function $v^{\varepsilon}(x)$ satisfy conditions $\left.\left.b_{1}\right)-b_{3}\right)$. Then the solution of problem (1)-(4) satisfies the estimates:

$$
\begin{aligned}
& \text { i. } \max _{0<t<T}\left(\left\|u^{\varepsilon}(x, t)\right\|_{L^{2}\left(\Omega^{\varepsilon}\right)}^{2}+\left\|\nabla_{x} u^{\varepsilon}(x, t)\right\|_{L^{2}\left(\Omega^{\varepsilon}\right)}^{2}\right) \leq C_{1}, \\
& \text { ii. } \max _{0<t<T}\left\|\frac{\partial u^{\varepsilon}(x, t)}{\partial t}\right\|_{L^{2}\left(\Omega^{\varepsilon}\right)}^{2}+\int_{0}^{T}\left\|\nabla_{x} \frac{\partial u^{\varepsilon}(x, t)}{\partial t}\right\|_{L^{2}\left(\Omega^{\varepsilon}\right)}^{2} d t \leq C_{2}, \\
& \text { iii. } \int_{0}^{T} \int_{\Omega^{\varepsilon}}\left(\frac{\partial u^{\varepsilon}(x, t+\Delta t)}{\partial t}-\frac{\partial u^{\varepsilon}(x, t)}{\partial t}\right)^{2} d x d t \leq C_{3} \Delta t .
\end{aligned}
$$

where the constants $C_{1}, C_{2}, C_{3}$ do not depend on $\varepsilon$.

P r o o f. The function $w^{\varepsilon}(x, t)=\frac{\partial u^{\varepsilon}(x, t)}{\partial t}$ is a solution of the following initial boundary-value problem:

$$
\begin{gathered}
\frac{\partial w^{\varepsilon}}{\partial t}-\Delta w^{\varepsilon}+\sum_{i=1}^{n} v_{i}^{\varepsilon}(x) \frac{\partial w^{\varepsilon}}{\partial x_{i}}=0, \text { in } \Omega^{\varepsilon} \times(0, T), \\
\frac{\partial w^{\varepsilon}}{\partial \nu}+\sigma_{u}^{\varepsilon}\left(x, u^{\varepsilon}\right) \cdot w^{\varepsilon}=0, \text { on } \partial F^{\varepsilon} \times(0, T), \\
w^{\varepsilon}(x, t)=0, \text { on } \partial \Omega \times(0, T), \\
w^{\varepsilon}(x, 0)=\Delta \varphi(x)-\sum_{i=1}^{n} v_{i}^{\varepsilon}(x) \frac{\partial \varphi(x)}{\partial x_{i}}, \text { in } \Omega^{\varepsilon} .
\end{gathered}
$$

We multiply (17) by $w^{\varepsilon}$ and integrate over the domain $\Omega^{\varepsilon}$. Then, integrating by parts and using the properties $\left.\left.b_{1}\right)-b_{3}\right)$, from (18)-(20) we obtain the relation

$$
\frac{1}{2} \cdot \frac{d}{d t}\left\|w^{\varepsilon}\right\|_{L^{2}\left(\Omega^{\varepsilon}\right)}^{2}+\left\|\nabla_{x} w^{\varepsilon}\right\|_{L^{2}\left(\Omega^{\varepsilon}\right)}^{2}+\int_{\partial F^{\varepsilon}} \sigma_{u}^{\varepsilon}\left(x, u^{\varepsilon}\right)\left|w^{\varepsilon}\right|^{2} d \Gamma=0 .
$$

Integrating the relation with respect to $t$ and using the property $a_{2}$ ) of the function $\sigma^{\varepsilon}(x, s)$, we have

$$
\left\|w^{\varepsilon}(x, t)\right\|_{L^{2}\left(\Omega^{\varepsilon}\right)}^{2}+\int_{0}^{t}\left\|\nabla_{x} w^{\varepsilon}\right\|_{L^{2}\left(\Omega^{\varepsilon}\right)}^{2} d t \leq\left\|w^{\varepsilon}(x, 0)\right\|_{L^{2}\left(\Omega^{\varepsilon}\right)}^{2} .
$$


From (20), taking into account property $b_{3}$ ) of the function $v^{\varepsilon}(x)$, we obtain

$$
\left\|w^{\varepsilon}(x, 0)\right\|_{L^{2}\left(\Omega^{\varepsilon}\right)} \leq\|\Delta \varphi\|_{L^{2}(\Omega)}+C\|\nabla \varphi\|_{L^{2}(\Omega)} .
$$

Using the properties of the function $\varphi(x)$, from $(21),(22)$ we get estimate 2 .

Estimate 1 is implied from estimate 2 and the inequality

$$
\|\psi(x, t)\|_{L^{2}\left(\Omega^{\varepsilon}\right)}^{2} \leq 2\|\psi(x, 0)\|_{L^{2}\left(\Omega^{\varepsilon}\right)}^{2}+2 T \int_{0}^{T}\left\|\frac{\partial \psi}{\partial t}\right\|_{L^{2}\left(\Omega^{\varepsilon}\right)}^{2} d t, \forall t \in[0, T] .
$$

Thus,

$$
\begin{gathered}
\left\|u^{\varepsilon}(x, t)\right\|_{L^{2}\left(\Omega^{\varepsilon}\right)}^{2}+\left\|\nabla_{x} u^{\varepsilon}(x, t)\right\|_{L^{2}\left(\Omega^{\varepsilon}\right)}^{2} \leq 2\left\|u^{\varepsilon}(x, 0)\right\|_{L^{2}\left(\Omega^{\varepsilon}\right)}^{2}+2\left\|\nabla u^{\varepsilon}(x, 0)\right\|_{L^{2}\left(\Omega^{\varepsilon}\right)}^{2} \\
+2 T(1+T) C_{2}=: C_{1} .
\end{gathered}
$$

Now we can get estimate 3 . Let us define

$$
\delta w^{\varepsilon}=w^{\varepsilon}(x, t+\Delta t)-w^{\varepsilon}(x, t) .
$$

Integrating (17) with respect to $t$ from $t$ to $t+\Delta t$, we have

$$
\delta w^{\varepsilon}-\Delta_{x}\left(\int_{t}^{t+\Delta t} w^{\varepsilon}(x, \tau) d \tau\right)+\sum_{i=1}^{n} v_{i}^{\varepsilon}(x) \frac{\partial}{\partial x_{i}} \int_{t}^{t+\Delta t} w^{\varepsilon}(x, \tau) d \tau=0 .
$$

We multiply (23) by $\delta w^{\varepsilon}$ and integrate over $\Omega^{\varepsilon}$. Then integrating by parts and using properties $\left.\left.b_{1}\right)-b_{3}\right)$, (18), we get

$$
\begin{aligned}
& \left\|\delta w^{\varepsilon}\right\|_{L^{2}\left(\Omega^{\varepsilon}\right)}^{2}+\int_{\partial F^{\varepsilon}}\left(\int_{t}^{t+\Delta t} \sigma_{u}^{\varepsilon}\left(x, u^{\varepsilon}\right) w^{\varepsilon}(x, \tau) d \tau\right) \delta w^{\varepsilon}(x, t) d \Gamma \\
& +\int_{\Omega^{\varepsilon}} \nabla_{x}\left(\int_{t}^{t+\Delta t} w^{\varepsilon}(x, \tau) d \tau\right) \nabla_{x} \delta w^{\varepsilon}(x, t) d x \\
& -\sum_{i=1}^{n} \int_{\Omega^{\varepsilon}}\left(v_{i}^{\varepsilon}(x) \int_{t}^{t+\Delta t} w^{\varepsilon}(x, \tau) d \tau \frac{\partial}{\partial x_{i}}\left(\delta w^{\varepsilon}(x, t)\right)\right) d x=0 .
\end{aligned}
$$

Integrating this equality with respect to $t$ from 0 to $T-\Delta$, applying the CauchyBunyakovsky inequality, taking into account property $b_{3}$ ) of the function $v^{\varepsilon}(x)$, 
and changing the order of integration in some integrals, we obtain

$$
\begin{aligned}
& \int_{0}^{T-\Delta}\left\|\delta w^{\varepsilon}\right\|_{L^{2}\left(\Omega^{\varepsilon}\right)}^{2} d t \leq\left|\int_{0}^{T-\Delta} \int_{\partial F^{\varepsilon}}\left(\int_{t}^{t+\Delta t} \sigma_{u}^{\varepsilon}\left(x, u^{\varepsilon}\right) w^{\varepsilon}(x, \tau) d \tau\right) \delta w^{\varepsilon} d \Gamma d t\right| \\
& +\Delta t\left(\int_{0}^{T}\left\|\nabla_{x} w^{\varepsilon}(x, \tau)\right\|_{L^{2}\left(\Omega^{\varepsilon}\right)}^{2} d \tau\right)^{1 / 2}\left(\int_{0}^{T-\Delta}\left\|\nabla_{x} \delta w^{\varepsilon}(x, t)\right\|_{L^{2}\left(\Omega^{\varepsilon}\right)}^{2} d t\right)^{1 / 2} \\
& +C \Delta t\left(\int_{0}^{T}\left\|w^{\varepsilon}(x, \tau)\right\|_{L^{2}\left(\Omega^{\varepsilon}\right)}^{2} d t\right)^{1 / 2}\left(\int_{0}^{T-\Delta}\left\|\nabla_{x} \delta w^{\varepsilon}(x, t)\right\|_{L^{2}\left(\Omega^{\varepsilon}\right)}^{2} d t\right)^{1 / 2} .
\end{aligned}
$$

It follows from the strong connectivity condition (7) that there exists a linear bounded operator $P^{\varepsilon}: H^{1}\left(\Omega^{\varepsilon}\right) \rightarrow H^{1}(\Omega)$. We define it more precisely. The function $\vartheta^{\varepsilon} \in H^{1}\left(\Omega^{\varepsilon}\right)$ can be extended in the whole domain $\Omega$ to the function $\tilde{\vartheta}^{\varepsilon}(x)=P^{\varepsilon} \vartheta^{\varepsilon}(x)$ in such a way that the extension $\tilde{\vartheta}^{\varepsilon}(x)$ satisfies the conditions: $\tilde{\vartheta}^{\varepsilon}(x) \in H^{1}(\Omega), \tilde{\vartheta}^{\varepsilon}(x)=\vartheta^{\varepsilon}(x)$ in $\Omega^{\varepsilon}$, and

$$
\int_{F^{\varepsilon}}\left(\left|\nabla \tilde{\vartheta}^{\varepsilon}\right|^{2}+\left|\tilde{\vartheta}^{\varepsilon}\right|^{2}\right) d x=\min _{\psi^{\varepsilon}=\vartheta^{\varepsilon}, x \in F^{\varepsilon}} \int_{F^{\varepsilon}}\left(\left|\nabla \psi^{\varepsilon}\right|^{2}+\left|\psi^{\varepsilon}\right|^{2}\right) d x .
$$

Thus, the extension $\tilde{\vartheta}^{\varepsilon}(x)$ is the solution of the problem

$$
\begin{aligned}
& \tilde{\vartheta}^{\varepsilon}(x)=\vartheta^{\varepsilon}(x), x \in \Omega^{\varepsilon} \cup \partial F^{\varepsilon}, \\
& -\Delta \tilde{\vartheta}^{\varepsilon}(x)+\tilde{\vartheta}^{\varepsilon}(x)=0, x \in F^{\varepsilon} .
\end{aligned}
$$

Since $u^{\varepsilon} \in W^{\varepsilon}$, then for almost all $t \in(0, T)$ the function $w^{\varepsilon}(x, t)=\frac{\partial u^{\varepsilon}}{\partial t} \in$ $H^{1}\left(\Omega^{\varepsilon}\right)$. Applying the operator $P^{\varepsilon}$ to the functions $u^{\varepsilon}(x, t), w^{\varepsilon}(x, t)$, we obtain the functions $\tilde{u}^{\varepsilon}(x, t)=P^{\varepsilon} u^{\varepsilon}(x, t), \tilde{w}^{\varepsilon}(x, t)=P^{\varepsilon} w^{\varepsilon}(x, t)$. By the uniqueness of the solution of problem (25), the derivative of the extended function and the extension of the derivative are the same, so we have $\tilde{w}^{\varepsilon}(x, t)=P^{\varepsilon} w^{\varepsilon}(x, t)=\frac{\partial \tilde{u}^{\varepsilon}}{\partial t}$.

Introduce a space $L^{2}\left(\Omega, \mu^{\varepsilon}\right)$ with measure $d \mu^{\varepsilon}=\hat{\sigma}^{\varepsilon}(x) d \Gamma$. In view of the generalized Sobolev theorem [19, p. 58] on the embedding of the space $H^{1}(\Omega)$ into the space $L^{2}\left(\Omega, \mu^{\varepsilon}\right)$ and by inequality (7), the following inequalities hold for each function $\vartheta^{\varepsilon} \in H^{1}\left(\Omega^{\varepsilon}\right)$ and its extension:

$$
\|\vartheta\|_{L^{2}\left(\Omega, \mu^{\varepsilon}\right)}=\|\tilde{\vartheta}\|_{L^{2}\left(\Omega, \mu^{\varepsilon}\right)} \leq C_{1}\|\tilde{\vartheta}\|_{H^{1}(\Omega)} \leq C_{2}\|\vartheta\|_{H^{1}\left(\Omega^{\varepsilon}\right)}
$$

where $C_{1}, C_{2}$ do not depend on $\varepsilon$.

Let us consider the first term in the right-hand side of inequality (24). Applying (26), the Cauchy-Bunyakovsky inequality and using the properties of $\sigma^{\varepsilon}(x, u)$, 
we obtain

$$
\begin{aligned}
& \left|\int_{0}^{T-\Delta} \int_{\partial F^{\varepsilon}}\left(\int_{t}^{t+\Delta t} \sigma_{u}^{\varepsilon}\left(x, u^{\varepsilon}\right) w^{\varepsilon}(x, \tau) d \tau\right) \delta w^{\varepsilon} d \Gamma d t\right| \\
& \leq \int_{0}^{T-\Delta} \int_{t}^{t+\Delta t}\left(\int_{\partial F^{\varepsilon}} \hat{\sigma}^{\varepsilon}(x)\left(w^{\varepsilon}(x, \tau)\right)^{2} d \Gamma\right)^{1 / 2}\left(\int_{\partial F^{\varepsilon}} \hat{\sigma}^{\varepsilon}(x)\left(\delta w^{\varepsilon}(x, t)\right)^{2} d \Gamma\right)^{1 / 2} d \tau d t \\
& =\int_{0}^{T-\Delta} \int_{t}^{t+\Delta t}\left\|w^{\varepsilon}(x, \tau)\right\|_{L^{2}\left(\Omega, \mu^{\varepsilon}\right)}\left\|\delta w^{\varepsilon}(x, t)\right\|_{L^{2}\left(\Omega, \mu^{\varepsilon}\right)} d \tau d t \\
& \leq C \int_{0}^{T-\Delta} \int_{t}^{t+\Delta t}\left\|w^{\varepsilon}(x, \tau)\right\|_{H^{1}\left(\Omega^{\varepsilon}\right)}\left\|\delta w^{\varepsilon}(x, t)\right\|_{H^{1}\left(\Omega^{\varepsilon}\right)} d \tau d t \\
& \leq C \Delta t^{1 / 2} \int_{0}^{T-\Delta}\left\|\delta w^{\varepsilon}(x, t)\right\|_{H^{1}\left(\Omega^{\varepsilon}\right)}\left(\int_{t}^{t+\Delta t}\left\|w^{\varepsilon}(x, \tau)\right\|_{H^{1}\left(\Omega^{\varepsilon}\right)}^{2} d \tau\right)^{1 / 2} d t \\
& \leq C \Delta t^{1 / 2}\left(\int_{0}^{T-\Delta}\left\|\delta w^{\varepsilon}(x, t)\right\|_{H^{1}\left(\Omega^{\varepsilon}\right)}^{2} d t\right)^{1 / 2}\left(\int_{0}^{T-\Delta} \int_{t}^{t+\Delta t}\left\|w^{\varepsilon}(x, \tau)\right\|_{H^{1}\left(\Omega^{\varepsilon}\right)}^{2} d \tau d t\right)^{1 / 2} .
\end{aligned}
$$

Changing the order of integration in the second term, we finally obtain

$$
\begin{aligned}
& \left|\int_{0}^{T-\Delta} \int_{\partial F^{\varepsilon}}\left(\int_{t}^{t+\Delta t} \sigma_{u}^{\varepsilon}\left(x, u^{\varepsilon}\right) w^{\varepsilon}(x, \tau) d \tau\right) \delta w^{\varepsilon} d \Gamma d t\right| \\
& \leq C \Delta t\left(\int_{0}^{T-\Delta}\left\|\delta w^{\varepsilon}(x, t)\right\|_{H^{1}\left(\Omega^{\varepsilon}\right)}^{2} d t\right)^{1 / 2}\left(\int_{0}^{T}\left\|w^{\varepsilon}(x, \tau)\right\|_{H^{1}\left(\Omega^{\varepsilon}\right)}^{2} d \tau\right)^{1 / 2} .
\end{aligned}
$$

From (24), (27) with the help of the first and second estimates, we obtain the third estimate of the lemma.

Lemma 1 is proved.

Lemma 2. Let the vector-function $v^{\varepsilon}(x)$ satisfy conditions $\left.\left.b_{2}\right)-b_{3}\right)$ and let the sequence of the vector-functions $\left\{v^{\varepsilon}(x)\right\}$ extended by zero into $F^{\varepsilon}$ converge weakly in $\left(L^{2}(\Omega)\right)^{n}$ to a vector-function $v(x)$ as $\varepsilon \rightarrow 0$. Let the function $\vartheta^{\varepsilon}(x)$ satisfy the inequality $\left\|\vartheta^{\varepsilon}\right\|_{H^{1}(\Omega)} \leq C$, where $C$ does not depend on $\varepsilon$, and let the sequence of the functions $\left\{\vartheta^{\varepsilon}(x)\right\}$ converge weakly in $H^{1}(\Omega)$ to a function $\vartheta(x) \in$ $H^{1}(\Omega)$ as $\varepsilon \rightarrow 0$. 
Then the sequence of the functions $\left\{\left(v^{\varepsilon}(x), \nabla \vartheta^{\varepsilon}(x)\right)\right\}$ converges weakly in $L^{2}(\Omega)$ to a function $(v(x), \nabla \vartheta(x))$ as $\varepsilon \rightarrow 0$.

P r o o f. Due to $\left.b_{3}\right)$ and the uniform boundedness of the norm $\left\|\vartheta^{\varepsilon}(x)\right\|_{H^{1}(\Omega)}$, the inequality

$$
\left\|\left(v^{\varepsilon}, \nabla \vartheta^{\varepsilon}\right)\right\|_{L^{2}(\Omega)} \leq C
$$

holds, where $C$ does not depend on $\varepsilon$.

It follows from the conditions of the lemma that the sequence of the vectorfunctions $v^{\varepsilon}(x)$, extended into $F^{\varepsilon}$ by zero, converges weakly in $\left(L^{2}(\Omega)\right)^{n}$ to a vector-function $v(x)$ as $\varepsilon \rightarrow 0$, and the sequence of the vector-functions $\nabla \vartheta^{\varepsilon}(x)$ converges weakly in $\left(L^{2}(\Omega)\right)^{n}$ to a vector-function $\nabla \vartheta(x)$ as $\varepsilon \rightarrow 0$. Furthermore, $\operatorname{div} v^{\varepsilon}(x)=0$ and $\operatorname{rot} \nabla \vartheta^{\varepsilon}(x)=0$. The lemma on the compensated compactness [26, p. 76] implies that the sequence of the functions $\left\{\left(v^{\varepsilon}(x), \nabla \vartheta^{\varepsilon}(x)\right)\right\}$ converges ${ }^{*}$ weakly in $L^{1}(\Omega)$ to the function $(v(x), \nabla \vartheta(x))$ as $\varepsilon \rightarrow 0$. From the ${ }^{*}$ weak convergence of the sequence $\left\{\left(v^{\varepsilon}(x), \nabla \vartheta^{\varepsilon}(x)\right)\right\}$ in $L^{1}(\Omega)$ and its uniform boundedness (28) in $L^{2}(\Omega)$ it follows [14, p.174] that $\left\{\left(v^{\varepsilon}(x), \nabla \vartheta^{\varepsilon}(x)\right)\right\}$ converges weakly in $L^{2}(\Omega)$ to the function $(v(x), \nabla \vartheta(x))$ as $\varepsilon \rightarrow 0$.

Lemma 2 is proved.

$\mathrm{R}$ e $\mathrm{m}$ a $\mathrm{r} \mathrm{k} 3$. In what follows, the extended solution $\tilde{u}^{\varepsilon}(x, t)$ of the initial boundary-value problem for each fixed $t \in(0, T)$ will be used as the function $\vartheta^{\varepsilon}(x)$ from Lemma 2.

\section{Proof of the Convergence Theorem}

We rewrite problem (1)-(3) for $\forall t \in(0, T)$ in the form

$$
\begin{gathered}
\Delta u^{\varepsilon}(x, t)=f^{\varepsilon}(x, t) \text { in } \Omega^{\varepsilon}, \\
\frac{\partial u^{\varepsilon}}{\partial \nu}+\sigma^{\varepsilon}\left(x, u^{\varepsilon}\right)=0 \text { on } \partial F^{\varepsilon}, \\
u^{\varepsilon}(x, t)=0 \text { on } \partial \Omega,
\end{gathered}
$$

where $f^{\varepsilon}(x, t)=\frac{\partial u^{\varepsilon}(x, t)}{\partial t}+\sum_{i=1}^{n} v_{i}^{\varepsilon}(x) \frac{\partial u^{\varepsilon}(x, t)}{\partial x_{i}}$.

In order to use Theorem 1 from [12], we first show that the sequence $f^{\varepsilon}(x, t)$ converges weakly in $L^{2}(\Omega)$ to some function $f(x, t)$ for almost all $t \in(0, T)$.

Let us extend the function $u^{\varepsilon}(x, t)$ to the whole domain $\Omega$ using the operator $P^{\varepsilon}(25): \tilde{u}^{\varepsilon}(x, t)=P^{\varepsilon} u^{\varepsilon}(x, t)$. Then $\frac{\partial u^{\varepsilon}(x, t)}{\partial t}=P^{\varepsilon} \frac{\partial u^{\varepsilon}(x, t)}{\partial t}=\frac{\partial \tilde{u}^{\varepsilon}(x, t)}{\partial t}$. By Lemma 1 , the functions $\tilde{u}^{\varepsilon}(x, t)$ and $\frac{\partial \tilde{u}^{\varepsilon}(x, t)}{\partial t}$ for all $t \in(0, T)$ satisfy the estimates:

$$
\max _{0<t<T}\left(\left\|\tilde{u}^{\varepsilon}(x, t)\right\|_{L^{2}(\Omega)}^{2}+\left\|\nabla_{x} \tilde{u}^{\varepsilon}(x, t)\right\|_{L^{2}(\Omega)}^{2}\right) \leq \tilde{C}_{1},
$$




$$
\begin{gathered}
\max _{0<t<T}\left\|\frac{\partial \tilde{u}^{\varepsilon}(x, t)}{\partial t}\right\|_{L^{2}(\Omega)}^{2}+\int_{0}^{T}\left\|\nabla_{x} \frac{\partial \tilde{u}^{\varepsilon}(x, t)}{\partial t}\right\|_{L^{2}(\Omega)}^{2} d t \leq \tilde{C}_{2}, \\
\int_{0}^{T} \int_{\Omega}\left(\frac{\partial \tilde{u}^{\varepsilon}(x, t+\Delta t)}{\partial t}-\frac{\partial \tilde{u}^{\varepsilon}(x, t)}{\partial t}\right)^{2} d x d t \leq \tilde{C}_{3} \Delta t,
\end{gathered}
$$

where the constants $\tilde{C}_{1}, \tilde{C}_{2}, \tilde{C}_{3}$ do not depend on $\varepsilon$.

It follows from (32), (33) that $\tilde{u}^{\varepsilon}(x, t) \in H^{1}(\Omega \times(0, T))$, and the norm $\left\|\tilde{u}^{\varepsilon}(x, t)\right\|_{H^{1}(\Omega \times(0, T))} \leq C$ is uniformly bounded with respect to $\varepsilon$. Thus the sequence $\left\{\tilde{u}^{\varepsilon}\right\}$ is weakly compact in $H^{1}(\Omega \times(0, T))$. Therefore, one can extract a subsequence $\left\{\tilde{u}^{\varepsilon_{k}}\right\}$ that converges weakly in $H^{1}(\Omega \times(0, T))$ and strongly in $L^{2}(\Omega \times(0, T))$ to some function $u(x, t) \in H^{1}(\Omega \times(0, T))$. Furthermore, the sequence $\left\{\nabla_{x} \tilde{u}^{\varepsilon_{k}}\right\}$ converges weakly in $\left(L^{2}(\Omega \times(0, T))\right)^{n}$ to a vector-function $\nabla_{x} u$.

It follows from (33) that the function $\frac{\partial \tilde{u}^{\varepsilon}(x, t)}{\partial t} \in L^{2}\left(0, T ; H^{1}(\Omega)\right)$, and the norm $\left\|\frac{\partial \tilde{u}^{\varepsilon}(x, t)}{\partial t}\right\|_{L^{2}\left(0, T ; H^{1}(\Omega)\right)} \leq C$ is uniformly bounded with respect to $\varepsilon$. Thus the sequence $\left\{\frac{\partial \tilde{u}^{k} k}{\partial t}\right\}$ is weakly compact in $L^{2}\left(0, T ; H^{1}(\Omega)\right)$. Then one can extract the subsequence $\left\{\frac{\partial \tilde{u}^{\varepsilon} \tilde{k}}{\partial t}\right\}$ that converges weakly in $L^{2}\left(0, T ; H^{1}(\Omega)\right)$ to the function $\frac{\partial u(x, t)}{\partial t} \in L^{2}\left(0, T ; H^{1}(\Omega)\right)$. In view of compact embedding $H^{1}(\Omega) \subset L^{2}(\Omega)$ and estimate (34), the subsequence $\left\{\frac{\partial \tilde{u}^{\varepsilon} \tilde{k}}{\partial t}\right\}$ converges strongly in $L^{2}(\Omega \times(0, T)$ ) (see [16, p. 225]) to the function $\frac{\partial u(x, t)}{\partial t} \in L^{2}\left(0, T ; H^{1}(\Omega)\right)$.

From the sequence $\left\{\varepsilon=\varepsilon_{\tilde{k}} \rightarrow 0\right\}$ we extract a subsequence $\left\{\varepsilon=\varepsilon_{\hat{k}} \rightarrow 0\right\}$ such that the subsequence $\left\{\tilde{u}^{\varepsilon} \hat{k}\right\}$ converges strongly in $L^{2}(\Omega)$ to the function $u(x, t)$, the subsequence $\left\{\nabla_{x} \tilde{u}^{\varepsilon_{\hat{k}}}\right\}$ converges weakly in $\left(L^{2}(\Omega)\right)^{n}$ to the vector-function $\nabla_{x} u$, and the subsequence $\left\{\frac{\partial \tilde{u}^{\hat{\varepsilon}} \hat{\hat{k}}}{\partial t}\right\}$ converges strongly in $L^{2}(\Omega)$ to the function $\frac{\partial u(x, t)}{\partial t}$ for almost all $t \in(0, T)$ (see [15, p. 407]).

It follows from condition 1 of Theorem 2 that the sequence of the characteristic functions $\left\{\chi^{\varepsilon}(x)\right\}$ of the domains $\Omega^{\varepsilon}$ converges weakly in $L^{2}(\Omega)$ to a function $b(x)$. On the other hand, the subsequence of the functions $\left\{\frac{\partial \tilde{u}^{\varepsilon} \hat{k}}{\partial t}\right\}$ converges strongly in $L^{2}(\Omega)$ to $\frac{\partial u(x, t)}{\partial t}$ for almost all $t \in(0, T)$. Therefore, $\left\{\chi^{\varepsilon_{\hat{k}}}(x) \frac{\partial \tilde{u}^{\varepsilon} \hat{k}(x, t)}{\partial t}\right\}$ converges weakly in $L^{2}(\Omega)$ to the function $b(x) \frac{\partial u(x, t)}{\partial t}$ for almost all $t \in(0, T)$.

Thus, in view of Lemma 2 , the sequence of the functions $f^{\varepsilon} \hat{k}(x)$ extended by zero into $F^{\varepsilon}$ converges weakly in $L^{2}(\Omega)$ as $\varepsilon_{\hat{k}} \rightarrow 0$ for almost all $t \in(0, T)$ to a function

$$
f(x, t)=b(x) \frac{\partial u(x, t)}{\partial t}+\sum_{i=1}^{n} v_{i}(x) \frac{\partial u(x, t)}{\partial x_{i}} .
$$

Now, using Theorem 1, we obtain from [12] that the solutions $u^{\varepsilon}(x, t)$ of problem (29)-(31) converge in $L^{2}\left(\Omega^{\varepsilon}, \Omega\right)$ as $\varepsilon=\varepsilon_{\hat{k}} \rightarrow 0$ to a function $u(x, t)$ satisfying equations (8), (9). Moreover, it follows from estimates (32), (33) that 
the function $\tilde{u}^{\varepsilon}(x, t)$ is continuous with respect to $t$ in a metric of $L^{2}(\Omega)$, uniformly with respect to $\varepsilon$. Thus we can conclude that $u(x, t) \in C\left(0, T ; L^{2}(\Omega)\right)$. It means that the limiting function $u(x, t)$ satisfies $u(x, 0)=\varphi(x)$, and it is the solution of the homogenized problem (8)-(10).

Let us show that the solution of the homogenized problem (8)-(10) is unique. Suppose that problem (8)-(10) has two different solutions $u_{1}(x, t)$ and $u_{2}(x, t)$. Then, in view of conditions of Theorem 2 and properties $\left.\left.b_{1}\right)-b_{3}\right)$ of the vectorfunction $v(x)$, we have

$$
\begin{aligned}
& \frac{d}{d t} \int_{\Omega} b(x)\left(u_{1}-u_{2}\right)^{2} d x=-2 \int_{\Omega} \sum_{i, k=1}^{n} a_{i k}(x) \frac{\partial\left(u_{1}-u_{2}\right)}{\partial x_{i}} \frac{\partial\left(u_{1}-u_{2}\right)}{\partial x_{k}} d x \\
& -\sum_{i=1}^{n} \int_{\Omega} v_{i}(x) \frac{\partial\left(u_{1}-u_{2}\right)^{2}}{\partial x_{i}} d x-\int_{\Omega}\left(c_{u}\left(x, u_{1}\right)-c_{u}\left(x, u_{2}\right)\right)\left(u_{1}-u_{2}\right) d x \\
& =-2 \int_{\Omega} \sum_{i, k=1}^{n} a_{i k}(x) \frac{\partial\left(u_{1}-u_{2}\right)}{\partial x_{i}} \frac{\partial\left(u_{1}-u_{2}\right)}{\partial x_{k}} d x \\
& -\int_{\Omega}\left(c_{u}\left(x, u_{1}\right)-c_{u}\left(x, u_{2}\right)\right)\left(u_{1}-u_{2}\right) d x \leq 0, \quad 0 \leq t \leq T .
\end{aligned}
$$

Thus the functions $u_{1}(x, t), u_{2}(x, t)$ are necessarily equal on $[0, T]$, and $u_{1}(x, 0)=$ $u_{2}(x, 0)$.

Since the solution to the homogenized problem is unique, then the whole sequence of the solutions $\left\{u^{\varepsilon}(x, t)\right\}$ of the initial problem converges in $L^{2}\left(\Omega^{\varepsilon}, \Omega\right)$ to the function $u(x, t)$ for almost all $t \in(0, T)$.

Theorem 2 is proved.

\section{References}

[1] N.S. Bakhvalov and G.N. Panasenko, Averaging Processes in Periodic Media. Mathematical Problems in the Mechanics of Composite Materials. Nauka, Moscow, 1984. (Russian)

[2] A. Beliaev, Homogenization of a Parabolic Operator with Signorini Boundary Conditions in Perforated Domains. - Asymptot. Anal. 40 (2004), 255-268.

[3] A. Bensoussan, J. Lions, and G. Papanicolaou, Asymptotic Analysis for Periodic Structures. North-Holland Publishing Company, Amsterdam-New York-Oxford, 1978.

[4] L.V. Berlyand and M.V. Goncharenko, Homogenization of the Diffusion Equation in Porous Media with Absorptions. - Teor. Funkts., Func. Analis i ikh Prilozhen. 52 (1989), 112-121. (Russian) 
[5] B. Cabarrubias and P. Donato, Homogenization of a Quasilinear Elliptic Problem with Nonlinear Robin Boundary Condition. - Appl. Anal.: An Intern. J. 91 (2012), No. 6, 1111-1127.

[6] B. Calmuschi and C. Timofte, Upscaling of Chemical Reactive Flows in Porous Media. iiCaius Iacobة̇ Conference on Fluid Mechanics and Texnical Appl., Bucharest (2005), 1-9.

[7] D. Cioranescu and J. Saint Jean Paulin, Homogenization of Reticulated Structures. Applied Mathematical Scienses, 136, Springer-Verlag, New York, 1999.

[8] D. Cioranescu and P. Donato, Homogenesation du Proble'me de Neumann non Homoge'ne dans des Ouverts Perfores. - Asymptot. Anal. 1 (1988), 115-138.

[9] D. Cioranescu, P. Donato, and R. Zaki, The Periodic Unfolding and Robin Problems in Perforated Domains. - C.R.A.S. Paris, Ser. 1342 (2006), 467-474.

[10] C. Conca, J. Diaz, and C. Timofte, Effective Chemical Processes in Porous Media. - Math. Models and Methods Appl. Sci. 13 (2003), No. 10, 1437-1462.

[11] C. Conca, J. Diaz, A. Linan, and C. Timofte, Homogenization in Chemical Reactive Floes. - Electron. J. Diff. Eq. 40 (2004), 1-22.

[12] M.V. Goncharenko and L.A. Khilkova, Homogenized Model of Diffusion in Porous Media with Nonlinear Absorption at the Boundary. - Ukr. Matem. Zhurn. 67 (2015), No. 9, 1201-1216. (Russian)

[13] M.V. Goncharenko and L.A. Khilkova, Homogenized Model of Diffusion in a LocallyPeriodic Porous Media with Nonlinear Absorption at the Boundary. - Dopovidi NANU 10 (2016), No. 6. (Russian)

[14] K. Iosida, Functional Analysis. Mir, Moscow, 1967. (Russian)

[15] A.N. Kolmogorov and S.V. Fomin, Elements of the Theory of Functions and Functional Analysis. Fizmatlit, Moscow, 2004. (Russian)

[16] O.A. Ladyzhenskaya, The Mathematical Theory of Viscous Incompressible Fluid. Nauka, Moscow, 1970. (Russian)

[17] O.A. Ladyzhenskaya, V.A. Solonnikov, and N.N. Ural'tseva, Linear and Quasilinear Equations of Parabolic Type. Nauka, Moscow, 1967. (Russian)

[18] V.A. Marchenko and E.Ya. Khruslov, Homogenized Models of MicroInhomogeneous Media. Naukova dumka, Kiev, 2005. (Russian)

[19] V.G. Maz'ya, Sobolev Spaces. Izdatel'stvo LGU, Leningrad, 1985. (Russian)

[20] T.A. Mel'nyk and D.Yu. Sadovyy, Homogenization of Quasilinear Parabolic Problem with Different Nonlinear Boundary Conditions Fourier Alternating in a Thick TwoLevel Junction of the Type 3:2:2. — Ukr. Matem. Zhurn. 63 (2011), No. 12, 16321656. (Ukrainian)

[21] T.A. Mel'nyk and O.A. Sivak, Asymptotic Analysis of a Boundary-Value Problem with the Nonlinean Multiphase Interactions in a Perforated Domain. - Ukr. Matem. Zhurn. 61 (2009), No. 4, 494-512. 
[22] T.A. Mel'nyk and O.A. Sivak, Asymptotic Analysis of a Parabolic Semilinear Problem with the Nonlinean Boundary Multiphase Interactions in a Perforated Domain. - J. Math. Sci. 164 (2010), No. 3, 1-27.

[23] T.A. Mel'nyk and O.A. Sivak, Asymptotic Approximations for Solutions to Quasilinear and Linear Parabolic Problems with Different Perturbed Boundary Conditions in Perforated Domains. - J. Math. Sci. 177 (2011), No. 1, 50-70.

[24] O.A. Oleinik, G.A. Yosifian, and A.S. Shamaev, Mathematical Problems in the Theory of Strongly Inhomogeneous Elastic Media. Izdatel'stvo MGU, Moscow, 1990. (Russian)

[25] A. Pankov, G-Convergence and Homogenization of Nonlinear Partial Differential Operators. Kluwer Academic Publishers, Dordrecht-Boston-London, 1997.

[26] A.L. Piatnitski, G.A. Chechkin, and A.S. Shamaev, Homogenization: Methods and Applications. Tamara Rozhkovskaya Press, Novosibirsk, 2007. (Russian)

[27] A. Piatnitski and V. Rybalko, Homogenization of Boundary Value Problems for Monotone Operators in Perforated Domains with Rapidly Oscillating Boundary Conditions of Fourier Type. - J. Math. Sci. 177 (2011), No. 1, 109-140.

[28] E. Sanchez-Palencia, Non-homogeneous Media and Vibration Theory. Lecture Notes in Physics 127. Springer-Verlag, New York, 1980.

[29] R.E. Showalter, Monotone Operators in Banach Space and NonlinearPartial Differential Equations. AMS, Providence, 1997.

[30] L. Tartar, The General Theory of Homogenization. A Personalized Introduction. Springer, Heidelberg-Dordrecht-London-New York, 2009.

[31] C. Timofte, Homogenization in Nonlinear Chemical Reactive Flows. Proc. of the 9th WSEAS Intern. Conference on Appl. Math., Istambul (2006), 250-255.

[32] C. Timofte, On the Homogenization of a Climatization Problem. - Studia Univ. iiBabes-Bolyai $\dot{z} \dot{\text { LII }}$ (2007), No. 2, 117-125.

[33] C. Timofte, Multiscale Analysis of Ionic Transport in Periodic Charged Media. Biomath 2 (2013), No. 2, 1-5.

[34] C. Timofte, N. Cotfas, and G. Pavel, On the Asymptotic Behaviour of Some Elliptic Problems in Perforated Domains. Romanian Reports in Phys. 64 (2012), No. 1, 514.

[35] V.V. Zhikov, S.M. Kozlov and O.A. Oleinik, Homogenization of Differential Operators. Fizmatlit, Moscow, 1993. (Russian) 